\title{
Morfoespecies de fitoplancton de la laguna el cometa en la Reserva de la Biosfera Pantanos de Centla
}

\section{Phytoplankton morphospecies of el cometa lagoon in the Reserva de la Biosfera Pantanos de Centla}

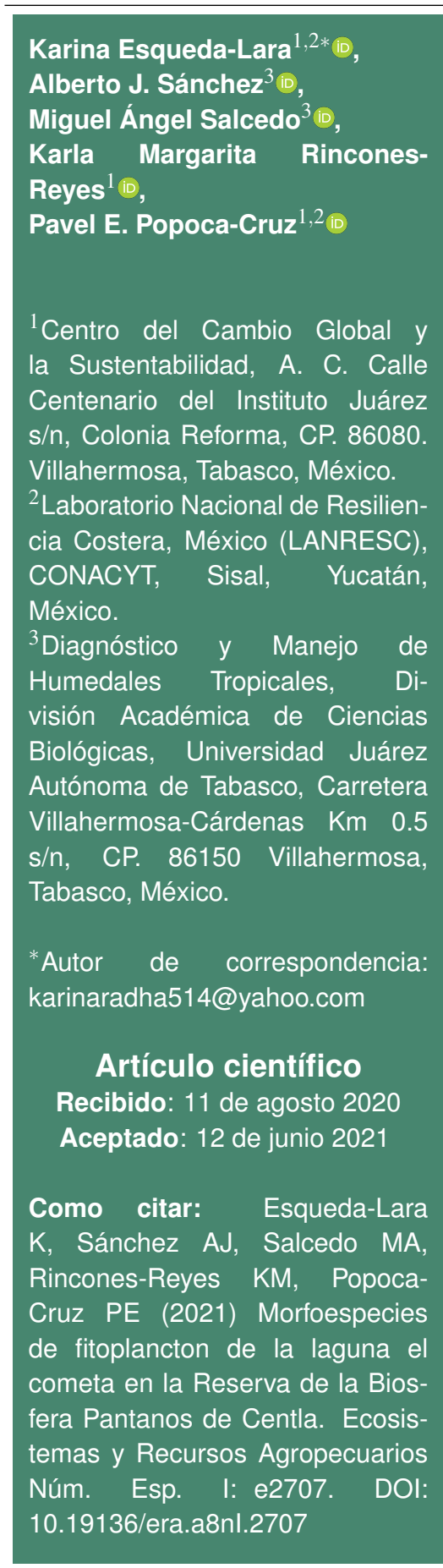

RESUMEN. La laguna El Cometa carece de información sobre la diversidad de microalgas, a pesar de ser el primer componente productivo de ecosistemas acuáticos e influir en la calidad y uso del agua. Por lo que se planteó el objetivo de determinar las morfoespecies de microalgas del fitoplancton en la laguna El Cometa así como, sus especies indicadoras y potencialmente tóxicas. Las muestras se recolectaron con una red de fitoplancton de 20 $\mu \mathrm{m}$ en seis sitios en la temporada de lluvias. Se registraron 50 taxones de seis grupos taxonómicos que representan los primeros registros para la Reserva de la Biosfera Pantanos de Centla. Treinta y tres de ellos se suman a los registros existentes en la planicie costera del río Usumacinta. Se encontraron cinco taxones marinos, siete potencialmente tóxicos y cinco bioindicadoras de contaminación y de condiciones de eutrofización que representan riesgo de salud pública.

Palabras clave: Cianotoxinas, eutrofización, microalgas.

ABSTRACT. El Cometa lagoon lacks information on microalgae diversity, despite its relevance as the first productive component of aquatic ecosystems and its influence on water quality. Therefore, the objective of determine the morphospecies of phytoplankton microalgae in El Cometa lagoon, as well as, their indicator species and the possible presence of potentially toxic species were raised. Phytoplankton samples with $20 \mu \mathrm{m}$ phytoplankton network were collected in six sites during rainy season. Fifty taxa from six taxonomic groups are the first records for the lagoon and Pantanos de Centla Biosphere Reserve. Thirty-three of them were added to the records for the coastal plain of the Usumacinta River. Five were marine, seven potentially toxic and five bioindicators of contamination and eutrophication conditions that represent a public health risk.

Key words: Cyanotoxin, Eutrophication, microalgae. 


\section{INTRODUCCIÓN}

En el territorio de la planicie costera de las cuencas de los ríos Grijalva-Usumacinta se localiza la Reserva de la Biosfera Pantanos de Centla (RBPC) y en su interior se encuentra la laguna El Cometa. La RBPC es un área prioritaria reconocida por RAMSAR y UNESCO que representa la mayor extensión de humedales en Mesoamérica y uno de los 15 humedales más importantes del mundo (LópezJiménez et al. 2020). Ésta incluye en su delta a los ríos San Pedro y San Pablo y el río GrijalvaUsumacinta que descargan sus aguas en el sureste del Golfo de México (RAMSAR 2001).

La Laguna El Cometa se ubica en una zona de gran diversidad en flora y fauna, por lo que es uno de los ecosistemas más representativos de la RBPC e incluso, en términos de plantas acuáticas es el área más importante de Mesoamérica (Jiménez-López et al. 2018, López-Jiménez et al. 2020). Sin embargo, la biodiversidad de microorganismos, en particular de las comunidades del fitoplancton, base de la cadena trófica en los ecosistemas acuáticos, ha sido escasamente estudiada en las cuencas de los ríos GrijalvaUsumacinta (Campos et al. 2012, Esqueda-Lara et al. 2016, Quiroz y Rivas 2017).

Las condiciones de relativa conservación que existen en la zona, así como las condiciones geográficas y climatológicas favorecen la presencia de los grupos taxonómicos como Bacillariophyta, Chlorophyta, Dinophyta, Euglenophyta y Ochrophyta que son comunes en zonas tropicales (Tolivia et al. 2008, Guamán-Burneo y González-Romero 2016). Las Cyanoprokaryota (Cyanophytas), también conocidas como cianobacterias, son organismos procariontes unicelulares y pluricelulares de morfología diversa, solitarias, coloniales o tricomas (filamentos), que se clasifican taxonómicamente tanto por la clasificación botánica (Cyanophytas) como por la nomenclatura microbiológica (Cyanoprokaryota). Son organismos fotosintéticos cuyos principales pigmentos son la clorofila a y las ficobiliproteínas, entre otros, que en su conjunto les permiten aprovechar un amplio espectro de longitud de onda (Lee 2008). Uno de los aspectos más importantes de las cianobac- terias es que muchos taxones tienen la capacidad de producir metabolitos secundarios, que pueden ser tóxicos para otros organismos dentro los ecosistemas (Stewart et al. 2006). De acuerdo con Lee (2008) a dichas toxinas se les conoce como cianotoxinas y de acuerdo con los órganos que dañan se conocen como hepatotoxinas para las que dañan células del hígado (microcistinas y nodularinas), neurotoxinas para las que afectan a la transmisión del impulso nervioso (saxitoxinas y anatoxinas) y dermatoxinas que afectan la piel (cilindrospermopsinas).

Por otro lado, las Euglenophyta es un grupo que se ha usado como indicador de ambientes eutróficos (Singh et al. 2013) y contaminados por heces y orina (Pereira y Azeiteiro 2003, Alves-da-Silva et al. 2010), mientras que las Bacillariophyta son usadas para la vigilancia de las condiciones ambientales de la calidad del agua (EPA 2002, Lane 2007). Las microalgas son productores primarios e indispensables para la conservación de las funciones de los ecosistemas acuáticos. Debido a que están vinculadas estrechamente a los ciclos biogeoquímicos y algunas variables físicas en la columna de agua (Arrigo 2005, Litchman et al. 2015). Por lo que muchas de las especies de este grupo heterogéneo se consideran bioindicadoras de la calidad del agua y de modificaciones de las condiciones hidrogeomorfológicas e hidrometereológicas (Zhao et al. 2013, Cruz-Ramírez et al. 2019), por lo que en lagunas permanentemente comunicadas con influencia marina estos organismos reflejan las condiciones ecológicas (Poot-Delgado et al. 2015). Por lo anterior el objetivo fue determinar las morfoespecies de microalgas del fitoplancton distribuidas en la laguna El Cometa de la RBPC, sus especies indicadoras de la influencia limnológicamarina y la posible presencia de especies potencialmente tóxicas que puedan dañar la biodiversidad acuática y terrestre o representar algún riesgo de salud pública.

\section{MATERIALES Y MÉTODOS}

\section{Área de estudio}

La Laguna El Cometa $\left(17^{\circ} 57^{\prime}-18^{\circ} 39^{\prime} \mathrm{N}\right.$ y $92^{\circ} 06^{\prime}-92^{\circ} 47^{\prime}$ W) ocupa un área de 67 hectáreas 
y está permanentemente interconectada con el río San Pedro y San Pablo a 22.5 km río arriba de la desembocadura de este río al sur del Golfo de México (Figura 1). Se localiza en la planicie de inundación en la cuenca del río Usumacinta, y debido a su altitud de $1 \mathrm{msnm}$, que es menor a la circundante, y a la pendiente del $1 \%$, funciona como nivel de base de las corrientes fluviales y de marea, por lo que permanece inundada con agua estuarina (Ramos-Reyes et al. 2016). La salinidad oscila entre 0.69 y 2.76 UPS y la fluctuación mínima de la temperatura del agua está entre 27.8 y $29^{\circ} \mathrm{C}$ (Montalvo et al. 2010). Derivado de sus condiciones topográficas, las depresiones del terreno presentan procesos acumulativos de turba superficial asociados con la descomposición de restos vegetales (Ramos-Reyes et al. 2016), originados por una extensa comunidad arbórea de Rhizophora mangle y Bucida buceras (Jiménez-López et al. 2018). La acumulación de la turba se evidencia en la Laguna El Cometa, ya que la relación entre los valores de la DBO5 y la DQO que se emplea como estimador de la biodegradación de los materiales orgánicos (Samudro y Mangkoedihardjo 2010), osciló entre 0 y 0.2 . No obstante, la laguna permanece comúnmente con saturaciones de oxígeno superiores al $30 \%$, en un intervalo de 18 a $122 \%$, valores menores a $1 \mathrm{mgL}^{-1}$ de las sales nitrogenadas inorgánicas y frecuentemente con valores menores que $0.1 \mathrm{mgL}^{-1}$ de fósforo total.

La vegetación circundante está dominada por Rhizophora mangle L. mezclada con selva mediana subperennifolia de Bucida buceras L. (Jiménez-López et al. 2017, López-Jiménez et al. 2020). Actualmente, la laguna es la segunda con mayor riqueza de manglar en México (Díaz-Jiménez 2007, JiménezLópez et al. 2017), con nueve especies de orquídeas (Jiménez-López y Domínguez Vázquez 2017) y 38 especies de epífitas. Por lo que la Laguna y sus alrededores es el área de mayor diversidad de este grupo de plantas (Jiménez-López et al. 2018).

\section{Recolección de muestras}

La recolecta del fitoplancton se realizó durante el mes de junio de 2014 en seis sitios dentro de la Laguna El Cometa, cinco cercanos a los diversos canales y uno al centro (Figura 2). Los muestreos se efectuaron de manera vertical con una red de $20 \mu \mathrm{m}$ de luz de malla a profundidad de $2 \mathrm{~m}$, y las se fijaron con formol al $4 \%$. Para luego analizarlas bajo dos microscopios ópticos (Carl Zeiss modelos AXIOLAB A1 y Primo Star), mediante las técnicas de campo claro, campo oscuro y contraste de fases. Para estimar la cantidad de alícuotas a revisar se calculó el área mínima de muestreo por medio de una curva de acumulación de especies (Brower et al. 1998). Las morfoespecies y sus variedades se identificaron con el uso de literatura especializada como Belcher y Swale (1976), De Souza Santos y Sant'Anna (2010), Komárek y Kovácik (1989), LópezMendoza et al. (2015), Ortega (1984), Sant'Anna et al. (2012), Rosini et al. (2013).

\section{Análisis de datos}

Para precisar las morfoespecies más importantes en las muestras obtenidas, la abundancia relativa de los organismos se estimó mediante el conteo de los primeros 300 organismos observados (McIntire y Overton 1971, Wetzel y Likens 2000). Por tratarse de abundancia relativa se contó como un solo organismo a los tricomas (filamentos) de Cyanoprokaryota. Se examinó la similitud en la distribución espacial de las morfoespecies, con los datos de presencia-ausencia por sitio se realizó un análisis de agrupamiento jerárquico, basado en la matriz de similitud con las medidas del índice de Jaccard (Legendre y Legendre 2003) bajo el modelo de enlace de promedio de grupo (Clarke y Gorley 2006). Además se aplicó la prueba de permutaciones (999) perfil de similitud (SIMPROF) para determinar diferencias en la posible composición de los grupos de las muestras con un nivel de significancia de $p<$ 0.05 (Clarke et al. 2008). Estos análisis se realizaron con el programa PRIMER v6. 1.6 (Clarke y Gorley 2006).

\section{RESULTADOS}

Un total de 50 morfoespecies, incluyendo variedades, fueron identificadas, de éstas, nueve tuvieron una determinación dudosa (Tabla 1). 


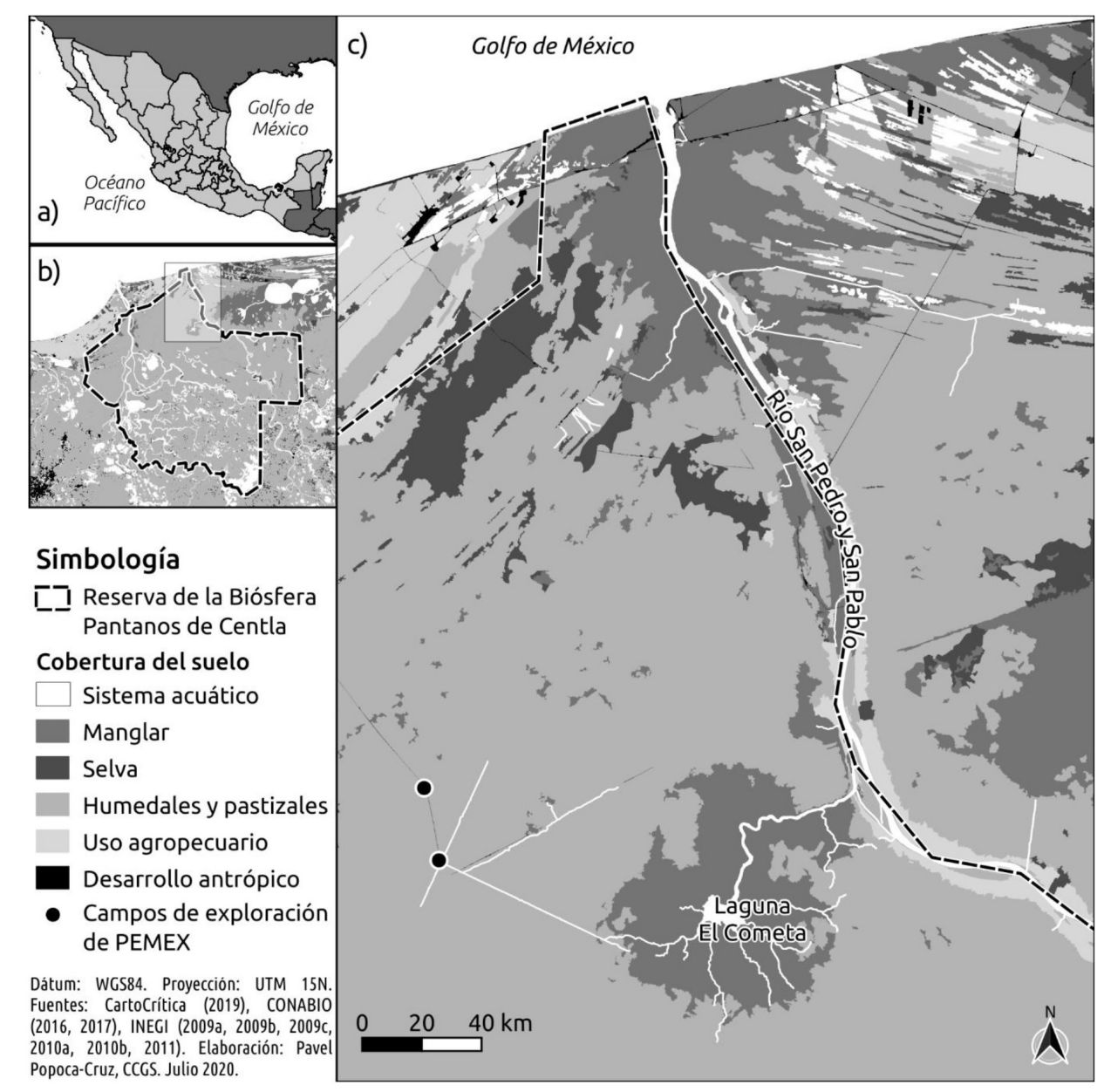

Figura 1. Ubicación y contexto ambiental de la Laguna El Cometa en México (a), dentro de la RBPC (b), y respecto al río San Pedro y San Pablo (c). Datos de CONABIO $(2016,2017)$ e INEGI $(2009 a, 2009 b, 2009$ c, 2010a, 2010b, 2011).

Las 50 morfoespecies pertenecen a seis grupos taxonómicos: Cyanoprokaryota (Cyanophyta), Bacillariophyta, Charophyta, Chlorophyta, Euglenophyta (Euglenozoa) y Ochrophyta. El total de estas morfoespecies representaron los primeros registros de fitoplancton para la RBPC y 33 de ellas (Tabla 1) se sumaron a los registros para la cuenca baja del río Usumacinta. El grupo más destacado por el número de especies fue Bacillariophyta con 22 taxones, seguido por Cyanoprokaryota con 11 taxones, mientras que, Ochrophyta fue el grupo con menos taxones, ya que sólo presentó uno. Entre las morfoespecies registradas se presentaron 36 dulceacuícolas, cinco marinas, dos salobres y siete de distribución amplia. Del grupo Cyanoprokaryota, siete de las 11 registradas fueron potencialmente nocivas (Tabla 1).

La riqueza específica por sitio de muestreo varió de 14 en el sitio E-6 a 23 en los sitios E-1 y E-3 (Figura 3). En todos los sitios se presentaron especies exclusivas. El sitio E-1 presentó, de sus 17 taxones registrados, tres que no se registraron en ningún otro sitio. El sitio E-2 presentó tres. Los sitios E-3 y E-4 cinco cada uno. El E-5 con tres y en el sitio E-6 solo un registro. En contraste, todos los sitios compartieron la presencia de Leptolyngbya tenuis y Cavinula maculata.

Las abundancias más representativas correspondieron a Leptolyngbya tenuis, que dominó con más del $50 \%$ en cinco de los sitios (E-1, E-2, E-3, 


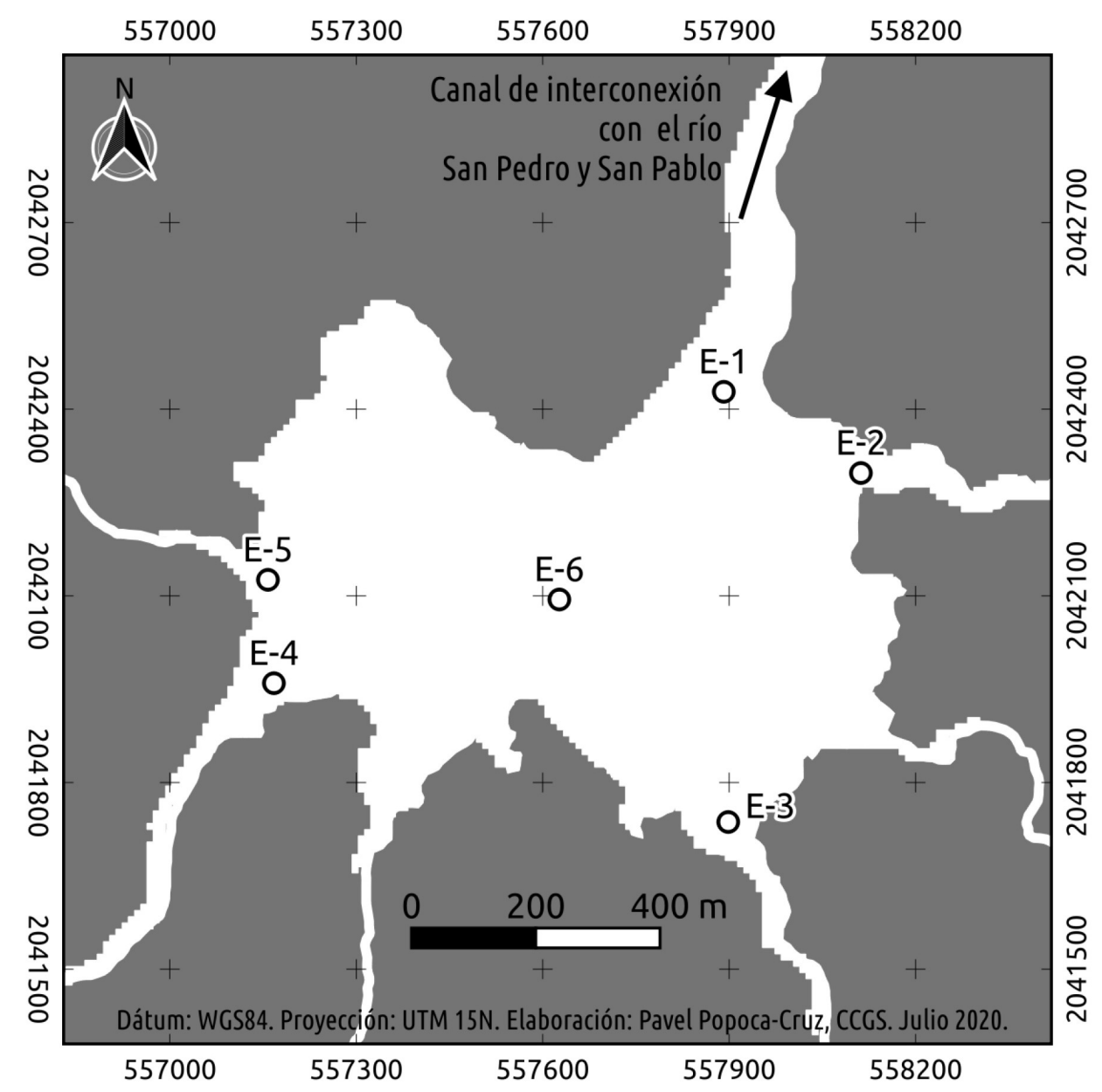

Figura 2. Ubicación de los sitios de muestreo de fitoplancton en la Laguna El Cometa en la Reserva de la Biosfera Pantanos de Centla. Elaboración propia.

E-4 y E-5), mientras que Geitlerinema sp. representó el $60.66 \%$ en el sitio E-6, y un $23.33 \%$ en el E-1. Esto fue seguido por las especies Cronbergia cf. planctonica y Pseudanabaena limnetica por contabilizar abundancias mayores al $20 \%$, C. cf. planctonica en E-3 y $P$. limnetica en los sitios E-4 y E-5 (Tabla 2).

De acuerdo con la distribución de las especies (presencia-ausencia) los grupos mostraron una tendencia heterogénea, ya que no se estructuraron grupos estadísticamente significativos $(p=0.085)$. La tendencia heterogénea entre grupos se debe a que la comunidad fitoplanctónica estuvo conformada, en gran parte, por diferentes especies en cada una de las estaciones de muestreo, pero los grupos de los sitios E-1, E-2, E-3 y E-6 fueron menos disímiles en- tre sí (Figura 4) al registrar valores del coeficiente de Jaccard (CJ) mayores que 0.32 (Tabla 3) y compartir seis taxones. El CJ con el valor más alto fue de 0.43 para los sitios E-1 y E-2 quienes compartieron 12 taxones. El siguiente valor de CJ (0.37) fue estimado para los sitios E-3 y E-6 con 10 taxones compartidos (Figura 4), donde sólo dos especies fueron exclusivas: Merismopedia elegans y Tryblionella scalaris. La variación del resto de los valores del CJ estuvo por debajo de 0.37 (Tabla 3). Dentro de los grupos distantes E-4 y E-5, el sitio E-5 fue más similar a los grupos E-1 y E-2 (CJ $\leq 0.3)$ que a los de E-3 y E-6 (CJ $\leq 0.25)$ (Tabla 3). Los sitios E-4 y E-5 compartieron siete especies, nueve con E-1 y 10 con E-2. Seguido de E-4 y E-5, las cuales compartieron cinco 
Tabla 1. Lista de las morfoespecies fitoplanctónicas en la laguna El Cometa en la Reserva de la Biosfera Pantanos de Centla.

\begin{tabular}{|c|c|c|c|}
\hline Especies por grupo taxonómico & limnetica & estuarina & marina \\
\hline \multicolumn{4}{|l|}{ Cyanoprokaryota } \\
\hline Cronbergia cf. planctonica Komárek & $\mathrm{x}$ & & \\
\hline +Geitlerinema sp. & $\mathrm{x}$ & & \\
\hline + Leptolyngbya tenuis (Gomont) Anagnostidis \& Komárek & $\mathrm{x}$ & & $x$ \\
\hline Merismopedia elegans A. Braun ex Kützing & $\mathrm{x}$ & $\mathrm{x}$ & $x$ \\
\hline Merismopedia tranquilla (Ehrenberg) Trevisan & $\mathrm{x}$ & & \\
\hline +Oscillatoria limosa C. Agardh ex Gomont & $\mathrm{x}$ & & \\
\hline +Planktothrix cf. compresa (Utermöhl) Anagnostidis y Komárek & $\mathrm{x}$ & & \\
\hline +Planktothrix rubescens (De Candolle ex Gomont) Anagnostidis \& Komárek & $\mathrm{x}$ & & \\
\hline +Planktothrix sp. & $\mathrm{x}$ & & \\
\hline +Pseudanabaena limnetica (Lemmermann) Komárek & $\mathrm{x}$ & & \\
\hline Spirulina sp. & $\mathrm{x}$ & & $\mathrm{x}$ \\
\hline \multicolumn{4}{|l|}{ Euglenophyta (Euglenozoa) } \\
\hline Lepocinclis acus (O. F. Müller) B. Marin \& Melkonian & $\mathrm{x}$ & & \\
\hline Lepocinclis oxyuris (Schmarda) B. Marin \& Melkonian & $\mathrm{x}$ & & \\
\hline Phacus acutus Pochmann & $\mathrm{X}$ & & \\
\hline Phacus curvicauda Svirenko & $\mathrm{x}$ & & $\mathrm{x}$ \\
\hline Phacus longicauda (Ehrenberg) Dujardin & $\mathrm{x}$ & & \\
\hline Phacus orbicularis Hübner & $\mathrm{x}$ & & \\
\hline \multicolumn{4}{|l|}{ Chlorophyta } \\
\hline Coeslastrum microporum Nägeli & $\mathrm{x}$ & & \\
\hline Monactinus simplex (Meyen) Corda & $\mathrm{x}$ & & \\
\hline Spyrogira sp. & $\mathrm{X}$ & & \\
\hline \multicolumn{4}{|l|}{ Charophyta } \\
\hline Closterium acutum Brébisson & $\mathrm{x}$ & & \\
\hline Closterium limneticum Lemmermann & $\mathrm{x}$ & & \\
\hline Closterium setaceaum Ehrenberg ex Ralfs & $\mathrm{x}$ & & \\
\hline Closterium venus Kützing ex Ralfs & $\mathrm{x}$ & & \\
\hline Cosmarium contractum $\mathrm{O}$. Kirchner & $\mathrm{x}$ & & \\
\hline Cosmarium formosulum Hoff & $\mathrm{X}$ & & \\
\hline Cosmarium quasillus P. Lundell & $\mathrm{X}$ & & \\
\hline \multicolumn{4}{|l|}{ Ochrophyta (Chrysophyceae) } \\
\hline Dinobryon sertularia Ehrenberg & $\mathrm{X}$ & & \\
\hline \multicolumn{4}{|l|}{ Bacillariophyta } \\
\hline Achnanthes inflata (Kützing) Grunow & $\mathrm{x}$ & & \\
\hline Actinoptychus senarius (Ehrenberg) Ehrenberg & $\mathrm{x}$ & & \\
\hline Amphora ovalis (Kützing) Kützing & $\mathrm{X}$ & & $\mathrm{x}$ \\
\hline Cavinula maculata (Bailey) J. Y. Li \& Y. Z. Qi & $\mathrm{X}$ & & \\
\hline Craticula ambigua (Ehrenberg) D.G. Mann & $\mathrm{x}$ & & \\
\hline Diploneis smithii var. rhombica Mereschkowsky & & & $\mathrm{x}$ \\
\hline Ditylum brightwellii (T. West) Grunow & & & $\mathrm{x}$ \\
\hline Fragilaria cf. capucina & $\mathrm{x}$ & & $\mathrm{x}$ \\
\hline Fragilaria virescens Ralf & $\mathrm{x}$ & & \\
\hline Gomphonema brasiliense Grunow & $\mathrm{X}$ & & \\
\hline Gomphonema sp. & $\mathrm{x}$ & & \\
\hline Gyrosigma attenuatum (Kützing) Rabenhorst & $\mathrm{x}$ & & \\
\hline Navicula palpebralis Brébisson ex W. Smith & & $\mathrm{X}$ & \\
\hline Nitzschia lanceolata W. Smith & & $\mathrm{X}$ & \\
\hline Nitzschia reversa W. Smith & $\mathrm{x}$ & & \\
\hline Placoneis gastrum (Eherenberg) Mereschkowsky & $\mathrm{x}$ & & \\
\hline Plagiotropis lepidoptera (W. Gregory) Kuntze & $\mathrm{x}$ & & $\mathrm{x}$ \\
\hline Pinnularia major (Kützing) Rabenhorst & $\mathrm{x}$ & & \\
\hline Pinnularia sp. & $\mathrm{x}$ & & \\
\hline Surirella striatula Turpin & & & $\mathrm{X}$ \\
\hline Tryblionella scalaris (Ehrenberg) Siver \& P. B. Hamilton & & & $\mathrm{x}$ \\
\hline Ulnaria ulna (Nitzsch) Compère & $\mathrm{x}$ & & \\
\hline
\end{tabular}




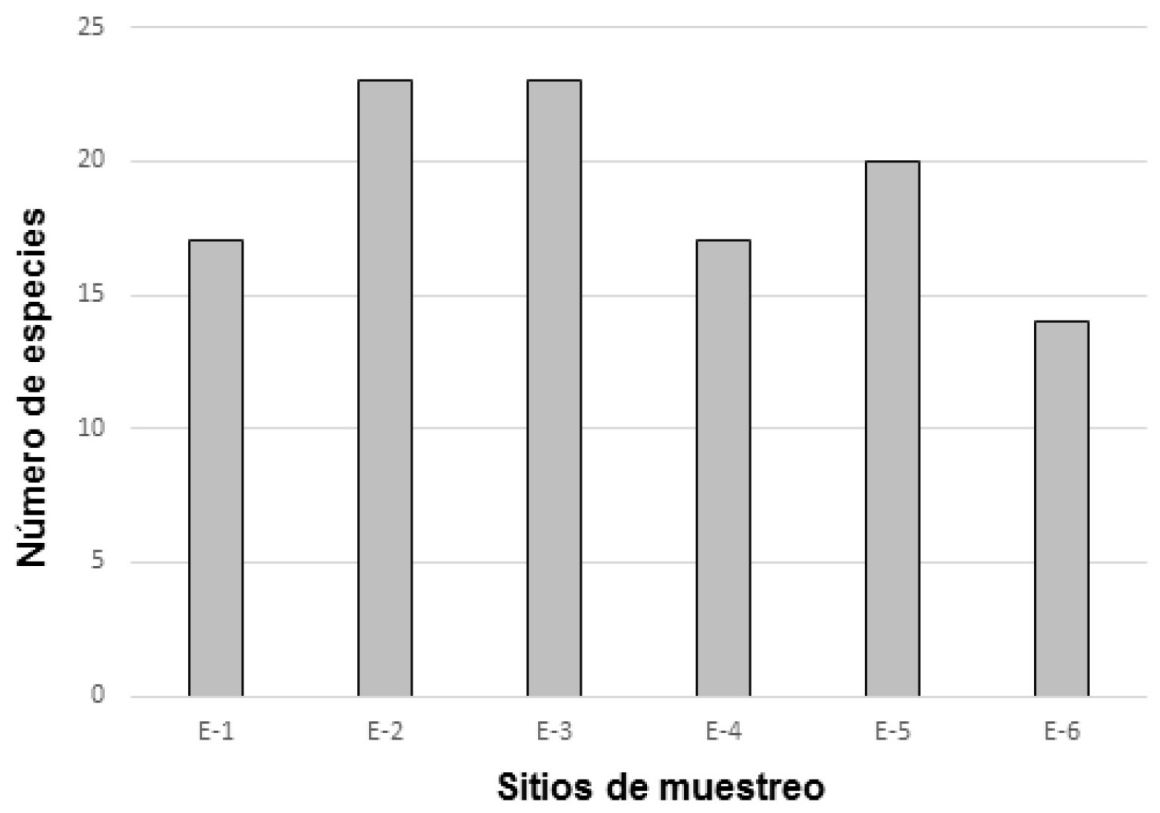

Figura 3. Riqueza específica por sitio de muestreo en la Laguna El Cometa en la Reserva de la Biosfera Pantanos de Centla.

especies, así como nueve con E-3 y siete con E6. El grupo del sitio E-4 fue el más diferente con CJ menores o iguales a 0.23 (Tabla 3), ya que presentó cinco especies exclusivas (Fragilaria cf. capucina, Lepocinclis oxyuris, Navicula palpebralis, Phacus longicauda y Surirella striatula); entre las cuales, tres de éstas presentaron abundancias relativas $(L$. oxyuris $(0.6 \%)$, P. longicauda (1.33\%), N. palpebralis $(0.33 \%)$ (Tabla 2). Por último, el valor mínimo de CJ fue entre los sitios E-4 y E-6, en los que únicamente coincidieron cuatro especies (Tabla 3 ).

\section{DISCUSIÓN}

Las 50 morfoespecies de los seis grupos taxonómicos (Cyanophyta (Cyanoprocaryota), Bacillariophyta, Charophyta, Chlorophyta, Euglenophyta (Euglenozoa) y Ochrophyta) se han registrado de manera común en ambientes oligohalinos y dulceacuícolas (Esqueda-Lara et al. 2016, Kufner y Giani 2017). De ellos, los grupos sobresalientes por sus riquezas fueron Bacillariophyta (44\%) y Cyanoprokaryota (22\%), lo que no es de ex- trañar, pues son grupos del fitoplancton con alta diversidad en los ambientes dulceacuícola y marino (Kooistra et al. 2007, Hernández-Becerril 2014).

Del total de las morfoespecies registradas en la laguna El Cometa, cinco fueron de distribución marina (Tabla 1) y a pesar de carecer de contenido celular al momento de su recolecta, son evidencia de la influencia de la marea en la zona. Los escasos registros de dichas especies marinas están asociados con el inicio de temporada de lluvias y su supervivencia pudo haber sido comprometida por el cambio de salinidad asociada con el incremento del flujo del río San Pedro y su conexión superficial lateral. Por lo que estas especies son una evidencia de la influencia de las corrientes de marea que penetran al menos $22.5 \mathrm{~km}$ de distancia de la línea de costa, principalmente, en el nivel mínimo del agua en la RBPC (Montalvo et al. 2010, Salcedo et al. 2012). Esta condición hidrológica es similar a la registrada en otras áreas prioritarias RAMSAR (Srichandan et al. 2019). Por lo que se espera que durante el menor caudal (temporada de secas) de los ríos de la cuenca del Usumacinta, en particular del río San Pedro y San Pablo, éstas y otras especies de microalgas marinas, 
Tabla 2. Distribución y abundancia relativa de las morfoespecies fitoplanctónicas en la laguna El Cometa en la Reserva de la Biosfera Pantanos de Centla.

\begin{tabular}{|c|c|c|c|c|c|}
\hline Morfoespecies & $\mathrm{E}-1$ & $\mathrm{E}-2$ & $E-3$ & $E-4$ & E-5 \\
\hline \multicolumn{6}{|l|}{ Cianoprokaryota } \\
\hline Cronbergia cf. planctonica & - & - & *27.33 & - & - \\
\hline Geitlerinema sp. & *23.33 & ${ }^{*} 0.33$ & - & - & - \\
\hline Leptolyngbya tenuis & *55.66 & *82.33 & *54.33 & ${ }^{*} 53.33$ & ${ }^{*} 68.33$ \\
\hline Merismopedia elegans & ${ }^{\star} 7.66$ & ${ }^{*} 0.33$ & * & - & - \\
\hline Merismopedia tranquilla & - & - & ${ }^{*} 0.33$ & - & - \\
\hline Oscillatoria limosa & - & * & *1.66 & - & - \\
\hline Planktothrix cf. compresa & *2 & - & - & - & - \\
\hline Planktothrix rubercens & - & - & - & - & ${ }^{* 1}$ \\
\hline Planktothrix sp. & - & - & * & ${ }^{*} 1.33$ & - \\
\hline Pseudanabaena limnetica & - & - & - & *32.33 & *27.33 \\
\hline Spirulina sp. & - & - & - & *9.33 & * \\
\hline \multicolumn{6}{|l|}{ Euglenophyta } \\
\hline Lepocinclis acus & * & * & - & - & - \\
\hline Lepocinclis oxyuris & - & - & - & ${ }^{*} 0.66$ & - \\
\hline Phacus acutus & * & ${ }^{*} 0.33$ & * & - & ${ }^{*} 0.33$ \\
\hline Phacus curvicauda & - & - & * & - & - \\
\hline Phacus longicauda & - & - & - & ${ }^{*} 1.33$ & - \\
\hline Phacus orbicularis & ${ }^{*} 0.33$ & - & - & - & - \\
\hline Chlorophyta & - & - & - & - & - \\
\hline Coeslastrum microporum & - & ${ }^{*} 0.33$ & * & - & - \\
\hline Monactinus simplex & - & * & * & * & - \\
\hline Spyrogira sp. & ${ }^{*} 6.66$ & ${ }^{*} 1.33$ & *6.66 & * & *0.66 \\
\hline Charophyta & - & - & - & - & - \\
\hline Closterium acutum & ${ }^{*} 0.33$ & - & - & - & - \\
\hline Closterium limneticum & - & * & - & - & ${ }^{*} 0.33$ \\
\hline Closterium setaceaum & - & - & ${ }^{*} 0.33$ & - & - \\
\hline Closterium venus & - & - & ${ }^{* 1}$ & - & *1 \\
\hline Cosmarium contractum & - & - & - & - & * \\
\hline Cosmarium formosulum & - & * & - & - & - \\
\hline Cosmarium quasillus & - & - & * & - & - \\
\hline \multicolumn{6}{|l|}{ Ochrophyta } \\
\hline \multicolumn{6}{|l|}{ Dinobryon sertularia } \\
\hline Ehrenberg & - & - & * & - & * \\
\hline \multicolumn{6}{|l|}{ Bacillariophyta } \\
\hline Achnanthes inflata (Kützing) Grunow & - & * & - & - & - \\
\hline Actinoptychus senarius & - & * & - & * & - \\
\hline Amphora ovalis & - & * & - & - & * \\
\hline Cavinula maculata & ${ }^{*} 0.66$ & ${ }^{*} 0.66$ & ${ }^{*} 0.33$ & *1.33 & * \\
\hline Craticulata ambigua & ${ }^{*} 1.33$ & ${ }^{*} 0.33$ & * & - & ${ }^{*} 0.33$ \\
\hline $\begin{array}{l}\text { Diploneis smithii var. } \\
\text { rhombica }\end{array}$ & ${ }^{*} 0.66$ & - & $\begin{array}{c}{ }^{*} 0.66 \\
0.33 \% \\
*\end{array}$ & $\stackrel{*}{*}{ }^{*} 1.33 \%$ & * \\
\hline Ditylum brightwellii & - & - & - & - & - \\
\hline Fragilaria cf capucina & - & - & - & * & - \\
\hline Fragilaria virescens & - & - & - & - & * \\
\hline Gomphonema brasiliense & - & - & * & - & - \\
\hline Gomphonema sp. & - & - & - & - & * \\
\hline Gyrosigma attenuatum & - & * & - & * & - \\
\hline Navicula palpebralis & - & - & - & ${ }^{\star} 0.33$ & - \\
\hline Nitzschia lanceolata & * & * & - & * & * \\
\hline Nitzschia reversa & * & - & - & - & * \\
\hline Placoneis gastrum & - & *1.33 & - & - & - \\
\hline Plagiotropis lepidoptera & ${ }^{*} 0.66$ & ${ }^{*} 0.33$ & ${ }^{*} 0.33$ & - & ${ }^{\star} 0.66$ \\
\hline Pinnularia major & * & * & ${ }^{*} 0.33$ & * & - \\
\hline Pinnularia sp. & - & * & - & - & * \\
\hline Surirella striatula & - & - & - & * & - \\
\hline
\end{tabular}

*Presencia del taxón, - ausencia del taxón, E-1 a E-5 = sitios de muestreo. 


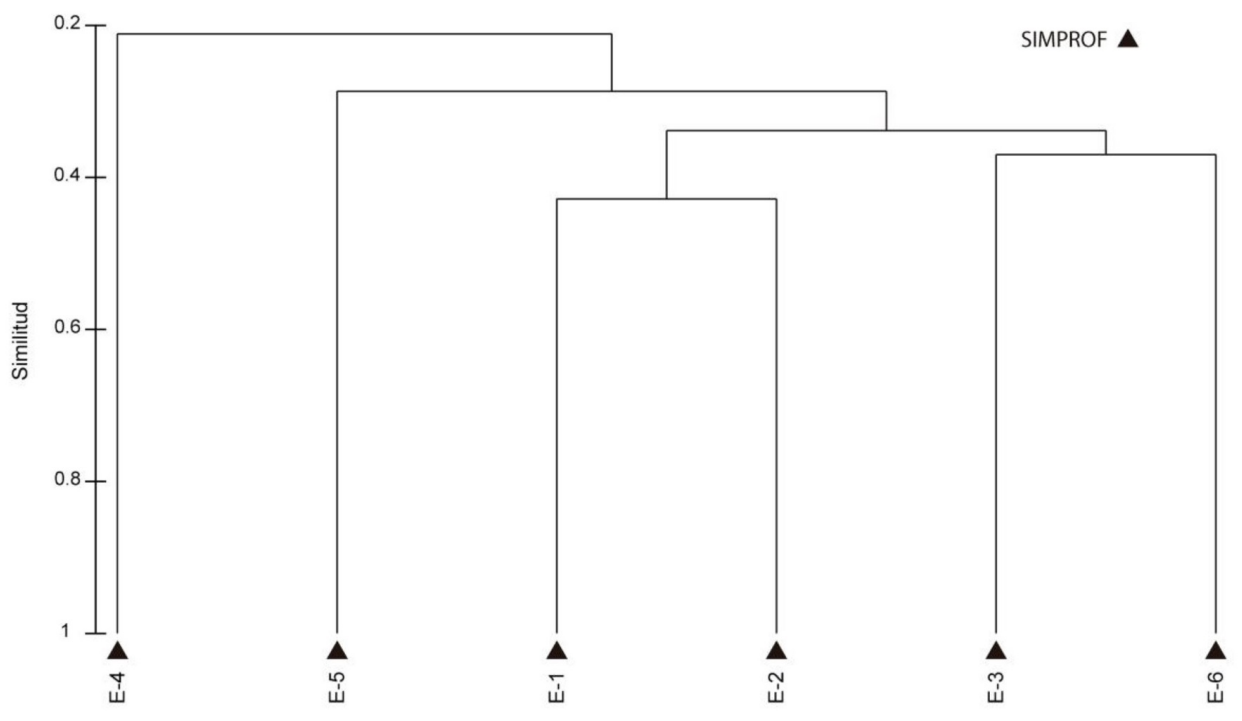

Figura 4. Similitud espacial de las agrupaciones fitoplanctónicas recolectadas en seis sitios de muestreo en la Laguna El Cometa, en la Reserva de la Biosfera Pantanos de Centla.

\begin{tabular}{|c|c|c|c|c|c|c|}
\hline & E1 & E2 & E3 & E4 & E5 & E6 \\
\hline 1 & 1 & 0.42857143 & 0.33333333 & 0.21428571 & 0.32142857 & 0.34782609 \\
\hline 2 & & 1 & 0.35294118 & 0.25 & 0.3030303 & 0.32142857 \\
\hline 3 & & & 1 & 0.21212121 & 0.26470588 & 0.37037037 \\
\hline 4 & & & & 1 & 0.23333333 & 0.14814815 \\
\hline \multirow[t]{2}{*}{5} & & & & & 1 & 0.25925926 \\
\hline & & & & & & 1 \\
\hline
\end{tabular}

se presenten con abundancias mayores a las registradas en este estudio.

Con respecto a los 11 taxones registrados de Cyanoprokaryota, al menos siete fueron potencialmente nocivas, es decir, con probable capacidad de producir metabolitos secundarios tóxicos, tanto para la fauna acuática como terrestre e incluso el ser humano (Stewart et al. 2006). De estos taxones, tres fueron de los géneros Geitlerinema y Planktothrix, los cuales contienen especies potencialmente productoras de microcistinas (Sivonen y Jones 1999, Bernard et al. 2011). Las morfoespecies $P$. rubescens y $P$. compressa también pueden producir microcistinas y en el caso de la segunda, además es capaz de producir anatoxinas (Sivonen y Jones 1999). L. tenuis produce las dos toxinas antes mencionadas, así como saxitoxinas (Mohamed y Alshehri 2015).

Otra especie productora de microcistina es Os- cillatoria limosa (Sivonen y Jones 1999). Dado a que las microcistinas son hepatotoxinas y que las anatoxinas y la saxitoxinas son neurotóxicas (Sivonen y Jones 1999), se considera que, tanto la fauna acuática como terrestre y en general la población humana que depende del agua del sistema para consumo directo y para preparar los alimentos, podrían estar expuestas al riesgo de intoxicaciones.

Otros taxones de Cyanoprokaryota que llaman la atención son $C$. cf. planctonica y Spirulina sp., debido a sus abundancias o características particulares. En el caso de $C$. cf. plantonica (27.33\%) es una especie de la cual se desconoce produzca toxina, pero es generadora de florecimientos algales nocivos que pueden inducir condiciones anóxicas en la columna de agua y con ello afectar a la fauna acuática (Chomérat et al. 2007). Con relación a Spirulina sp., este género es reconocido por la producción de sus- 
tancias bioactivas (Mohamed y Al-Shehri 2015).

Algunas especies de los grupos Chlorophyta

y Euglenophyta son consideradas indicadoras de aguas contaminadas y eutrofizadas (Singh et al. 2013). Entre las halladas se registran las especies Monactinus simplex (Meyen) Coda, Phacus curvicauda Svirenko y Phacus orbicularis K. Hübner. Entre estas, $M$. simplex está asociada con ecosistemas eutróficos y con ambientes con descargas de aguas residuales (Singh et al. 2013). En tanto que, especies del género Phacus son abundantes en ambientes eutróficos (Singh et al. 2013) altamente contaminados por heces y orina (Pereira y Azeiteiro 2003).

Las morfoespecies registrados en cada sitio no presentaron grupos significativos estadísticamente, pero se distinguieron dos asociaciones, una formada por cuatro sitios (E-1, E-2, E-3 y E-6) al este y dos sitios diferentes (E-4 y E-5) de la anterior agrupación al oeste de la laguna. Los cuatro sitios aglomerados que se ubican al este de la laguna reciben mayor influencia del canal de interconexión con el río San Pedro y San Pablo, mientras que los otros dos sitios (E-4 y E-5) están en el margen oeste de la laguna (Figura 2) que recibe la influencia de los campos de exploración de PEMEX por medio de un canal comunicado que descarga en una dendrita de la laguna EI Cometa. Este posible patrón temporal de distribución espacial causado por influencia antropogénica necesita ser corroborado, pero se sustenta en la presencia exclusiva de las especies $F$. cf. capucina, L. oxyuris, $N$. palpebralis, P. longicauda y $S$. striatula en E4 y Cosmarium contractum, Fragilaria virescens y Ghomphonema sp. en E-5, además de que el $40 \%$ de las morfoespecies se presentaron solo en uno de los sitios y en que únicamente el $4 \%$ de las especies estuvieron presentes en todos los sitios. Aunque no se consideró evaluar el efecto de la marea, la huella que dejan los registros de las especies marinas del fitoplancton, muestra la influencia de la misma sobre la composición de dicha comunidad, así como el impacto posible asociado con la entrada de aguas provenientes de la zona de exploración de PEMEX y la escorrentía implícita de este ecosistema acuático. Así lo sugieren, además de la presencia de especies marinas, como diatomeas y la ausencia de dinoflage- lados.

La RBPC ha presentado un intenso cambio de uso de suelo por las prácticas agropecuarias y un escaso manejo de residuos sólidos y líquidos (Sánchez et al. 2007, De la Rosa-Velázquez et al. 2017). Estas prácticas han favorecido tanto la contaminación por agroquímicos en la zona (Sánchez y Barba 2005, Córdoba-Carrillo et al. 2010, Barba-Macías et al. 2018), como la posible presencia de especies potencialmente tóxicas y nocivas. Por lo mismo, se considera impostergable amortiguar o detener el cambio de uso de suelo y la aplicación de agroquímicos para evitar la reproducción súbita (florecimiento algal) de las Cyanoprokaryota potencialmente tóxicas y nocivas, que pueden afectar de manera crítica la vida de la fauna y la salud humana.

Las 50 morfoespecies representan los primeros registros de fitoplancton para la RBPC y 33 de ellas se suman a los registros para la cuenca baja del río Usumacinta (Esqueda-Lara et al. 2016). Estas especies pertenecen a los tres grupos más representativos del fitoplancton e incluye especies potencialmente tóxicas, indicadoras de contaminación por carga orgánica y fecal y condiciones eutrofizadas. En el caso de la Laguna El Cometa su presencia es esperable debido a que las condiciones topográficas y de altitud en depresión propician los procesos acumulativos de turba superficial asociados con la descomposición de restos vegetales (Ramos-Reyes et al. 2016). Estas condiciones de acumulación de materia orgánica y especies indicadoras de condiciones tóxicas sumadas a la posible influencia del campus PEMEX a través del canal que drena directamente a la E4, abre la hipótesis a resolver sobre una condición ecológica perturbada que propicia vulnerabilidad para la población humana que usa de manera directa el agua del río.

El posible escenario de vulnerabilidad de las poblaciones asentadas en la Laguna El Cometa requiere de la estimación de la abundancia e identificación de las algas, sus abundancias y cepas potencialmente tóxicas en la RBPC, ya que hasta el 2000, el $75 \%$ de los pobladores de la RBPC dependían de forma directa del agua de estos ecosistemas para satisfacer sus necesidades básicas (Sánchez et al. 
2007) y que en la actualidad este uso directo del agua es evidente.

\section{CONCLUSIONES}

Los estudios dedicados a las microalgas, con especial énfasis en las potencialmente tóxicas y las indicadoras de condiciones ecológicas perturbadas son indispensables para estimar la vulnerabilidad del ecosistema y de los asentamientos humanos que dependen de éste y en especial de su agua para su higiene y consumo directo.

\section{AGRADECIMIENTOS}

Al financiamiento para el trabajo de campo del proyecto FOMIX (Tab-2012-c28-194316) y para análisis el proyecto FORDECYT 273646 "Cambio global y sustentabilidad en la Cuenca del Usumacinta y zona marina de influencia: Bases para la adaptación al cambio climático desde la cuenca y la gestión territorial". A Fátima del Rosario Antonio Arias, Arisbeth Hernández Alonso y Allan Keith Cruz Ramírez, por el apoyo técnico.

\section{LITERATURA CITADA}

Alves-da-Silva SM, Pereira VC, Moreira CS, Friedrich F (2010) O gênero Phacus (Euglenophyceae) em lago urbano subtropical, no Jardim Botânico de Porto Alegre, Rio Grande do Sul, Brasil. Acta Botanica Brasilica 15: 713-726.

Arrigo KR (2005) Marine microorganisms and global nutriente cycles. Nature 437: 349-355.

Barba-Macías E, Mesa-Jurado A, Espinoza-Tenorio A, Ortega-Argueta A (2018) Biodiversity conservation in the Pantanos de Centla Biosphera Reserve: Ecological and Socioeconomic Threats. In: Ortega-Rubio A (eds.) Mexican natural resources management and biodiversity conservation. Springer Nature. Switzerland. pp: $1-40$.

Bernard C, Froscio S, Campbell R, Monis P, Humpage A, Fabbro L (2011) Novel toxic effects associated with a tropical Limnothrix/Geitlerinema-like cyanobacterium. Environmental Toxicology 26: 260-270.

Belcher H, Swale E (1976) A beginner's guide to freshwater algae. Institute of Terrestrial Ecology, Her Majesty's Stationery Office. Londres, Reino Unido. 47p.

Brower JE, Zar JH, von Ende CN (1998) Field and laboratory methods for general ecology. McGraw-Hill. Boston MA, Estados Unidos. 273p.

Campos B, Custodio TN, Torres C, Rivas MG, Cruz L (2012) Registro preliminar de la composición fitoplanctónica de la laguna de Mecoacán, Paraíso, Tabasco, México. Kuxulkab’ 18: 65-72.

Chomérat N, Garnier R, Bertrand C, Cazaubon A (2007) "Seasonal succession of cyanoprokaryotes in a hypertrophic oligo-mesohaline lagoon from the South of France". Estuarine Coastal Shelf Science. 72: 591-602.

Clarke KR, Gorley RN (2006) PRIMER v6: User manual/tutorial. PRIMER-E Ltd. Plymouth, UK. 190p.

Clarke KR, Somerfield PJ, Gorley RN (2008) Testing of null hypotheses in exploratory community analyses: similarity profiles and biota-environment linkage. Journal of Experimental Marine Biology and Ecology 366: 56-69.

Cruz-Ramírez AK, Salcedo MA, Sánchez AJ, Barba E, Mendoza JD (2019) Relationship among physicochemical conditions, chlorophyll-a concentration, and water level in a tropical river-floodplain system. International Journal Environmental Science Technology 16: 3869-3876. 
CONABIO (2016) Conjunto de datos vectoriales del mapa de uso del suelo y vegetación de la zona costera asociada a los manglares en la Región Golfo de México en 2015. Escala: 1:50,000. 1a Edición. Comisión Nacional para el Conocimiento y Uso de la Biodiversidad. Sistema de Monitoreo de los Manglares de México (SMMM). Ciudad de México, México. http://www.conabio.gob.mx/informacion/metadata/gis/gm_oc 2015gw.xml?_httpcache=yes\&_xsl=/db/metadata/xsl/fgdc_html.xsl\&_indent=no. Fecha de consulta: 05 octubre 2017.

CONABIO (2017) Conjunto de datos ráster de la Cobertura de tipos de vegetación y uso de suelo MADMex 31 Clases, 2015 (satélite RapidEye). Comisión Nacional para el Conocimiento y Uso de la Biodiversidad. Monitoring Activity Data for the Mexican REDD+ program (MAD-Mex). Ciudad de México. https://monitoreo.conabio.gob.mx/snmb_charts/descarga_datos_madmex.html. Fecha de consulta: 05 octubre 2017.

Córdoba-Carrillo A, Pérez-Sánchez E, Rodríguez-Quevedo F, Ovando-Hidalgo N, Zequeira-Larios C (2010) Agroquímicos utilizados en la Reserva de la Biosfera Pantanos de Centla: Una afectación indirecta para el Cocodrilo de Pantano (Crocodylus moreletii) Kuxulkab' 17: 31-42.

De la Rosa-Velázquez MI, Espinoza-Tenorio A, Díaz-Perera MA, Ortega-Argueta A, Ramos-Reyes R, Espejel I (2017) Development stressors are stronger than protected area management: A case of the Pantanos de Centla Biosphere Reserve, Mexico. Land Use Policy 67: 340-351.

De Souza Santos KR, Sant'Anna CL (2010) Cianobactérias de diferentes tipos de lagoas "salina", "salitrada" e "baía" representativas do Pantanal da Necholândia, MS, Brasil. Brazilian Journal of Botany 33: 61-83.

Díaz-Jiménez P (2007) Flora epífita de los manglares de Tabasco, México. Kuxulkab' 25: 11-17.

EPA (2002) Methods for evaluating wetland condition: using algae to assess environmental conditions in wetlands, Office of Water, U.S. Environmental Protection Agency Washington, DC. EPA-822-R-02-021. Washington, DC. $42 p$.

Esqueda-Lara K, Sánchez AJ, Valdés-Lagunes G, Salcedo MA, Franco-Torres AE, Florido R (2016) Fitoplancton en el humedal tropical Chaschoc en la cuenca baja del río Usumacinta. Revista Mexicana de Biodiversidad 87: 1177-1188.

Guamán-Burneo MC, Gónzalez-Romero NP (2016) Microalgas y cianobacterias encontradas en sistemas lacustres de áreas protegidas de Los Andes y Amazonía del Ecuador. Quito, Ecuador. 147p.

Hernández-Becerril DU (2014) Biodiversidad de algas planctónicas marinas (Cyanobacteria, Prasinophyceae, Euglenophyta, Chrysophyceae, Dictyochophyceae, Eustigmatophyceae, Parmophyceae, Raphidophyceae, Bacillariophyta, Cryptophyta, Haptophyta, Dinoflagellata) en México. Revista Mexicana de Biodiversidad, Supl. 85: 44-53.

INEGI (2009a) Conjunto de datos vectoriales de la carta topográfica E15B62b. Instituto Nacional de Estadística y Geografía. Aguascalientes, México. https//www.inegi.org.mx/app/biblioteca/ficha.html?upc=702825721725. Fecha de consulta: 06 de septiembre de 2020.

INEGI (2009b) Conjunto de datos vectoriales de la carta topográfica E15B62c. Instituto Nacional de Estadística y Geografía. Aguascalientes, México. https//www.inegi.org.mx/app/biblioteca/ficha.html?upc=702825721732. Fecha de consulta: 06 de septiembre de 2020.

INEGI (2009c) Conjunto de datos vectoriales de la carta topográfica E15B62f. Instituto Nacional de Estadística y Geografía. Aguascalientes, México. https//www.inegi.org.mx/app/biblioteca/ficha.html?upc=702825721763. Fecha de consulta: 06 de septiembre de 2020. 
INEGI (2010a) Conjunto de datos vectoriales de la carta topográfica E15B62e. Instituto Nacional de Estadística y Geografía. Aguascalientes, México. https//www.inegi.org.mx/app/biblioteca/ficha.html?upc=702825721756. Fecha de consulta: 06 de septiembre de 2020.

INEGI (2010b) Conjunto de datos vectoriales de la carta topográfica E15B72b. Instituto Nacional de Estadística y Geografía. Aguascalientes, México. https//www.inegi.org.mx/app/biblioteca/ficha.html?upc=702825722043. Fecha de consulta: 06 de septiembre de 2020.

INEGI (2011) Conjunto de datos vectoriales de la carta topográfica E15B72c. Instituto Nacional de Estadística y Geografía. Aguascalientes, México. https//www.inegi.org.mx/app/biblioteca/ficha.html?upc=702825551384. Fecha de consulta: 06 de septiembre de 2020.

Jiménez-López DA, Domínguez-Vázquez MA (2017) Lista comentada de las orquídeas de la laguna El Cometa, Tabasco, México. Orquideología 4: 80-91.

Jiménez-López DA, Roblero-Velasco RJ, Martínez- Meléndez N, Ocampo G, Gallardo-Cruz JA (2017) Relación entre las variables del forofito y la riqueza de epífitas vasculares en los Pantanos de Centla, Tabasco, México. Acta Botánica Mexicana 121: 125-137.

Jiménez-López DA, Peralta-Carreta C, Solórzano JV, Cervantes-Jiménez GL, Domínguez-Vázquez MA (2018) A checklist of vascular epiphytes of El Cometa Lagoon, Pantanos de Centla Biosphere Reserve, Mexico. Journal of Threatened Taxa 10: 12589-12597.

Komárek J, Kovácik L (1989) Trichome structure of four Aphanizomenon taxa (Cyanophyceae) from Czechoslovakia, with notes on the taxonomy and delimitation of the genus. Plant Systematics and Evolution 164: 47-64.

Kooistra WHCF, Gersonde R, Medlin LK, Mann DG (2007) The origin and evolution of the diatoms: their adaptation to a planktonic existence. In: Falkowsky PG, Knoll AH (eds.) Evolution of primary producers in the sea. Elsevier Academic Press, Singapore. pp: 207-249.

Kufner DCL, Giani A (2017) Euglenophyta de lagoas da região da Nhecolândia, Pantanal Sul-Matogrossense, Brasil. Hoehnea 44: 277-294.

Lane CR (2007) Assessment of isolated wetland condition in Florida using epiphytic diatoms at genus, species, and subspecies taxonomic resolution. EcoHealth 4: 219-230.

Lee RE (2008) Phycology. 4a ed. Cambridge University Press. Nueva York. 547p.

Legendre P, Legendre L (2003) Numerical ecology. 2nd ed. Elsevier Science, Amsterdam, Holanda. 853p.

Litchman E, de Tezanos Pinto P, Edwards KF, Klausmeier CA, Kremer CT, Thomas MK (2015) Global biogeochemical impacts of phytoplankton: a trait-based perspective. Journal of Ecology 103: 1384-1396.

López-Mendoza Z, Tavera R, Novelo E (2015) El fitoplancton de un canal de Xochimilco y la importancia de estudiar ecosistemas acuáticos urbanos. Revista especializada en Ciencias Químico-Biológicas 18: 13-28.

López-Jiménez LN, Jiménez-López DA, Castillo-Acosta OC, Gallardo-Cruz JA, Fernández-Montes de Oca AI (2020) Plantas vasculares de la Reserva de la Biosfera Pantanos de Centla, México. Botanical Sciences 98: 159-204.

McIntire CD, Overton WS (1971) Distributional patterns in assemblages of attached diatoms from Yaquina Estuary, Oregon. Ecology 52: 758-777.

Mohamed ZA, AI-Shehri AM (2015) Biodiversity and toxin production of cyanobacteria in mangrove swamps in the Red Sea off the southern coast of Saudi Arabia. Botanica Marina 58: 23-34. 
Montalvo H, Sánchez AJ, Florido R, Macossay-Cortez A (2010) Lista de crustáceos distribuidos en troncos hundidos en el humedal tropical Pantanos de Centla, al sur del Golfo de México. Revista Mexicana de Biodiversidad 81: S121- S131.

Ortega M (1984) Catálogo de algas continentales recientes de México. Universidad Nacional Autónoma de México. Distrito Federal, México. 566p.

Pereira MJ, Azeiteiro UMM (2003) Ecological notes on the species of Phacus Dujardin (Euglenophyta) from the central region of Portugal. Acta Oecologica 24: 33-48.

Poot-Delgado CA, Okolodkov YB, Aké-Castillo JA, Rendón-von Osten J (2015) Annual cycle of phytoplankton with emphasis on potentially harmful species in oyster beds of Términos Lagoon, southeastern Gulf of Mexico 50: 465-477.

Quiroz N, Rivas MG (2017) Euglenoideos en dos lagunas urbanas en Villahermosa. Kuxulkab' 23: 35-40.

Ramos-Reyes R, Zavala-Cruz J, Gama-Campillo LM, Pech-Pool D, Ortiz-Pérez MA (2016) Indicadores geomorfológicos para evaluar la vulnerabilidad por inundación ante el ascenso del nivel del mar debido al cambio climático en la costa de Tabasco y Campeche, México. Boletín de la Sociedad Geológica Mexicana 68: 581-598.

RAMSAR (2001) Ficha informativa de los humedales de RAMSAR, Reserva de la biosfera Pantanos de Centla. CONANP. https://rsis.ramsar.org/RISapp/files/RISrep/MX733RIS.pdf. Fecha de consulta: 10 de marzo de 2020.

Rosini EF, Sant'Anna CL, Tucci A (2013) Cyanobacteria de pesqueiros da região metropolitana de São Paulo, Brasil. Rodriguésia 64: 399-417.

Salcedo MA, Sánchez A, de la Lanza G, Kampichler C, Florido R (2012) Condición ecológica del humedal tropical Pantanos de Centla. En: Sánchez AJ, Chiappa-Carrara X, Brito R (eds.) Recursos Acuáticos Costeros del Sureste. CONCIYTEY. Mérida, Yucatán, México. pp: 112-136.

Samudro G, Mangkoedihardjo S (2010) Review on BOD and COD ratio: Triangle zone for toxic, biodegradable and stable levels. International Journal of Academic Research 2: 235-239.

Sánchez AJ, Barba E (2005) Biodiversidad de Tabasco. En: Bueno J, Álvarez F, Santiago S (eds.) Biodiversidad del Estado de Tabasco. CONABIO - Instituto de Biología, UNAM. México. pp: 1-16.

Sánchez AJ, Salcedo MA, Florido R, Armenta A, Rodríguez-Leal C, Galindo A, Mogel E (2007) Pantanos de Centla, un humedal costero tropical. En: de la Lanza G, Hernández-Pulido S (eds.) Las aguas interiores de México. Conceptos y casos. AGT Editor. México. pp: 399-422.

Sant'Anna CL, Tucci A, Azevedo MTP, Melcher SS, Werner VR, Malone CFS, Rossini EF, Jacinavicius FR, Hentschke GS, Osti JAS, Santos KRS, Gama-Jünior WA, Rosal C, Adame G (2012) Atlas de cianobacterias e microalgas de águas continentais brasileiras. Publicação electrónica, Instituto de Botânica, Núcleo de Pesquisa em Ficologia. https://www.infraestruturameioambiente.sp.gov.br/institutodebotanica/wpcontent/uploads/sites/235/2013/09/virtuais_3atlas.pdf. Fecha de consulta: 13 de septiembre de 2020.

Srichandan S, Baliarsingh SK, Prakash S, Lotliker AA, Parida C, Sahu KC (2019) Spatiotemporal distribution and composition of phytoplankton assemblages in a coastal tropical lagoon: Chilika, India. Environmental Science and Pollution Research 26: 12025-12041.

Singh UB, Ahluwalia AS, Sharma C, Jindal R, Thakur RK (2013) Planktonic indicator: A promising tool for monitoring water quality (early-warning signals). Ecology, Environmental and Conservation 19: 793-800. 
Sivonen K, Jones G (1999) Toxic Cyanobacteria in water: A guide to their public health consequences, monitoring and management. In: Chorus A, Bartram B (eds.) Cyanobacterial toxins. F \& FN Spon. Great Britain. pp: 1-70.

Tolivia A, Boltovskoy A, Barón EJ, Marciales LM, González-González LM, Núñez M, Vigna MS, Echenique RO, Sala SE, Conforti V (2008) Microalgas acuáticas: la otra escala de la biodiversidad en la Amazonía colombiana. Instituto Amazónico de Investigaciones Científicas Sinchi. Bogotá, Colombia. 240p.

Stewart I, Webb PM, Schluter PJ, Shaw G (2006) Recreational and occupational field exposure to freshwater cyanobacteria-a review of anecdotal and case reports, epidemiological studies and the challenges for epidemiologic assessment. Environmental Health: A Global Access Science Source 5: 6.

Wetzel GR, Likens EG (2000) Limnological analyses. 4th Edition. Springer-Verlag. Nueva York. 429p.

Zhao Z, Mi T, Xia L Yan W, Jiang Y, Gao Y (2013) Understanding the patterns and mechanisms of urban water ecosystem degradation: phytoplankton community structure and water quality in the Qinhuai River, Nanjing City, China. Environmental Science and Pollution Research 20: 5003-5012. 